\title{
Brain grey matter abnormalities in medication-free patients with major depressive disorder: a meta-analysis
}

\author{
Y.-J. Zhao' ${ }^{1}$, M.-Y. Du ${ }^{1}$ t, X.-Q. Huang ${ }^{1}$, S. Lui ${ }^{1}$, Z.-Q. Chen ${ }^{1}$, J. Liu ${ }^{1}$, Y. Luo ${ }^{1}$, X.-L. Wang ${ }^{1}$, \\ G. J. Kemp ${ }^{2}$ and Q.-Y. Gong ${ }^{1 *}$ \\ ${ }^{1}$ Huaxi MR Research Center (HMRRC), Department of Radiology, West China Hospital of Sichuan University, Chengdu, People's Republic of China \\ ${ }^{2}$ Magnetic Resonance and Image Analysis Research Centre (MARIARC) and Institute of Ageing and Chronic Disease, University of Liverpool, \\ Liverpool, UK
}

\begin{abstract}
Background. Because cerebral morphological abnormalities in major depressive disorder (MDD) may be modulated by antidepressant treatment, inclusion of medicated patients may have biased previous meta-analyses of voxel-based morphometry (VBM) studies. A meta-analysis of VBM studies on medication-free MDD patients should be able to distinguish the morphological features of the disease itself from those of treatment.
\end{abstract}

Method. A systematic search was conducted for the relevant studies. Effect-size signed differential mapping was applied to analyse the grey matter differences between all medication-free MDD patients and healthy controls. Meta-regression was used to explore the effects of demographics and clinical characteristics.

Results. A total of 14 datasets comprising 400 medication-free MDD patients and 424 healthy controls met the inclusion criteria. The pooled meta-analysis and subgroup meta-analyses showed robustly reduced grey matter in prefrontal and limbic regions in MDD. Increased right thalamus volume was only seen in first-episode medication-naive patients, and increased grey matter in the bilateral anterior cingulate cortex only in medication wash-out patients. In meta-regression analyses the percentage of female patients in each study was negatively correlated with reduced grey matter in the right hippocampus.

Conclusions. By excluding interference from medication effects, the present study identified grey matter reduction in the prefrontal-limbic network in MDD. The subgroup meta-analysis results suggest that an increased right thalamus volume might be a trait directly related to MDD, while an increased anterior cingulate cortex volume might be an effect of medication. The meta-regression results perhaps reveal the structural underpinning of the sex differences in epidemiological and clinical aspects of MDD.

Received 5 June 2013; Revised 23 January 2014; Accepted 13 February 2014; First published online 21 March 2014

Key words: Effect size signed differential mapping, grey matter, magnetic resonance imaging, major depressive disorder, meta-analyses, voxel-based morphometry.

\section{Introduction}

Major depressive disorder (MDD) (lifetime prevalence $13-16 \%)$ is a complex constellation of disturbances in cognition, behaviour and physical functioning (Rizvi \& Kennedy, 2012). MDD is the third leading cause of global disease burden and a leading cause of disability worldwide (Mathers et al. 2008). Depression is clinically and aetiologically heterogeneous (Hasler, 2010). A major research effort aims to elucidate its biological underpinnings, and thus to develop more effective treatments.

\footnotetext{
* Address for correspondence: Q.-Y. Gong, M.D., Huaxi MR Research Center (HMRRC), Department of Radiology, West China Hospital of Sichuan University, No. 37 Guo Xue Xiang Chengdu, 610041, People's Republic of China.

(Email: qiyonggong@hmrrc.org.cn)

t These authors contributed equally to this work.
}

Numerous structural magnetic resonance imaging (MRI) studies have sought to identify the key brain areas involved in the pathogenesis of depressive symptoms. Voxel-based morphometry (VBM) is a fully automated method for analysing neuromorphological MRI data which allows unbiased investigation of differences in brain structure between patients and healthy subjects (Ashburner \& Friston, 2001). It has accuracy comparable with manual volumetry (Davies et al. 2009) and overcomes the technical limitations of region-of-interest approaches. VBM has been widely used in characterizing subtle changes in brain structure in a variety of neurological and psychiatric diseases. VBM studies have reported that MDD is associated with widespread local abnormalities in many brain regions, such as the frontal gyrus (Wagner et al. 2008; Scheuerecker et al. 2010; Salvadore et al. 2011), anterior cingulate gyrus (Tang et al. 2007; Wagner et al. 2008; Salvadore et al. 2011) and hippocampus 
(Wagner et al. 2008; Zou et al. 2010; Arnone et al. 2012). However, these reports are inconsistent in detail, probably because of small sample sizes, heterogeneous subjects and methodological differences. Identifying consistent results from VBM studies of MDD patients through meta-analysis is therefore of particular importance.

New analytical tools have made possible wholebrain meta-analyses using the spatial coordinates of reported structural imaging findings to produce consensus neuroanatomical maps of abnormalities. Three recent meta-analyses of VBM studies found that MDD was characterized by volume reductions in the bilateral anterior cingulate cortex (ACC) (Bora et al. 2012; Du et al. 2012; Lai, 2013), right middle and inferior frontal gyrus (Du et al. 2012), parahippocampal gyrus and hippocampus (Du et al. 2012). However, most of the patients included in these were receiving antidepressant drugs at the time of scanning. It is recognized that the inclusion of medicated patients may bias meta-analyses and limit the interpretability and generalizability of the results.

Animal and human studies yield primary evidence on the effect of therapy on grey matter. For example, 3 weeks of agomelatine administration affects neurogenesis in the dorsal and ventral hippocampus of adult rats (Banasr et al. 2006); similarly, 4 weeks of tianeptin administration protects against the decreased hippocampal volume produced by stress in tree shrews (Czeh et al. 2001). Medication-related differences in regional brain volumes have been reported in patients with mood disorders (Brambilla et al. 2002; Chang et al. 2005; Lavretsky et al. 2005; Savitz et al. 2010; Smith et al. 2013). Adult patients with MDD show increased grey matter in the left dorsolateral prefrontal cortex (DLPFC) after 12 weeks of selective serotonin reuptake inhibitor (SSRI) treatment (Smith et al. 2013), while in geriatric MDD patients antidepressant exposure is associated with increased orbitofrontal grey matter (Lavretsky et al. 2005). In addition, a meta-analysis has reported that the greater the proportion of medicated individuals in the depressed sample, the larger is the abnormality in amygdala volume (Hamilton et al. 2008). Furthermore, there is growing evidence that decreased levels of brain-derived neurotrophic factor and other neurotrophic factors in MDD could contribute to the atrophy of limbic structures such as the hippocampus and prefrontal cortex, and that the neurotrophic actions of antidepressants could reverse neuronal atrophy and cell loss (Duman \& Monteggia, 2006). It is therefore conceivable that antidepressant medication itself could cause regional grey matter volume changes in MDD. Thus studies of unmedicated patients could better elucidate brain abnormalities that are directly related to the disease itself.
Direct study of unmedicated depressed patients faces substantial practical and ethical problems (Blease, 2011). Although published VBM studies on medication-free MDD patients have recently become sufficiently numerous to warrant specific metaanalysis, no such meta-analysis of grey matter volumes has yet been published. Therefore, the goals of this study were twofold: first, to establish the most prominent and replicable grey matter abnormalities in medication-free MDD patients; and, second, to examine the effects of demographics and clinical characteristics on grey matter volumes in MDD, using a new coordinate-based meta-analytic technique called effectsize signed differential mapping (ES-SDM). ES-SDM combines several useful features of earlier methods, such as activation likelihood estimation and multilevel kernel density analysis. It also introduces some novel improvements: to avoid any voxel appearing significant in opposite directions, both positive and negative differences are reconstructed in the same signed differential map; the use of effect sizes allows combination of reported peak coordinates with statistical parametric maps, thus allowing more detailed and accurate meta-analyses; lastly, complementary analyses such as jack-knife, subgroup and meta-regression analyses are readily used to assess the robustness and heterogeneity of the results (Radua et al. 2012b).

\section{Method}

\section{Inclusion of studies}

A systematic strategy was used to search for relevant studies published in Pubmed, Embase, Web of Science and Science Direct up to March 2013, using the keywords 'depression' or 'depressive disorder' or 'unipolar depression' plus 'voxel-based morphometry' or 'VBM' or 'voxel"' or 'morphometry'. The reference lists of these studies were checked to identify further studies for inclusion. Studies were included according to the following criteria: (1) used VBM to analyse whole-brain grey matter changes in MDD patients; (2) compared MDD patients with healthy control subjects; and (3) investigated medication-free MDD patients, who either had never received antidepressive medications (i.e. were medication-naive), or who underwent a wash-out period before MRI scanning. Findings based on small volume correction were excluded. Theoretical papers and reviews were excluded, as well as studies that reported adolescent or late-life depression. For studies where multiple independent patient samples were compared with healthy controls, the appropriate coordinates were included as separate datasets. For studies using overlapping samples, the study with the most subjects was included. 


\section{Recorded variables}

For each included study we recorded: numbers, sex and mean age of subjects; illness duration, depression symptom severity and mean numbers of episodes; drug status; the percentage of patients who had ever received psychotherapy; the statistical threshold of the main findings, and the method employed to correct whole-brain results for multiple comparisons.

\section{Meta-analysis of regional differences in grey matter}

Regional differences in grey matter were analysed using ES-SDM (http://www.sdmproject.com), a voxelbased meta-analytic approach that uses the reported peak coordinates to re-create maps of the effect size of group differences in grey matter. For peak coordinates, the re-creation is based on first converting the peak $t$ value to Hedges' effect size and then applying a non-normalized Gaussian kernel to the voxels close to the peak. In ES-SDM both positive and negative differences are reconstructed in the same map, which prevents a particular voxel appearing to be significant in opposite directions. Importantly, negative studies are also included in the meta-analysis. Jack-knife sensitivity analysis was used to test the replicability of results and meta-regression methods to characterize the impact of key clinical variables on brain structure (Radua et al. 2012b).

ES-SDM has been described in detail elsewhere (Radua et al. 2011, 2012a,b) and applied to metaanalysis studies on neuropsychiatric disorders including schizophrenia and bipolar disorder (Bora et al. 2011; Palaniyappan et al. 2012). Here we summarize its main features. First, we ensured that the same threshold was used throughout the whole brain in each included study (although not necessarily the same in different studies), in order to avoid biases towards liberally thresholded brain regions (Radua \& Mataix-Cols, 2009). Second, an effect-size signed map of the differences in grey matter was separately recreated for each study. Third, the mean map was obtained by calculating the mean of the study maps, weighted by the inverse of each study variance and accounting for inter-study heterogeneity. Statistical significance was assessed by a permutation test (Radua et al. 2012b).

\section{Sensitivity analysis}

A systematic whole-brain voxel-based jack-knife sensitivity analysis was used to test the replicability of the results. This involves repeating the main analysis 14 times, discarding a different study each time: if a brain region remains significant in all or most of the combinations of studies it can be concluded that this finding is highly replicable.

\section{Subgroup analysis}

We conducted a subgroup analysis of first-episode medication-naive MDD patients. Strategically, recruiting such individuals helps to reduce confounders such as illness duration and previous antidepressant treatment that may dilute the neuropathological findings in MDD. Because medication withdrawal will not immediately reverse any medication-related grey matter changes, we compared these results with a subgroup analysis of MDD patients who underwent a wash-out period before MRI scanning.

\section{Meta-regression analysis}

The following variables were explored by metaregression: the percentage of female MDD patients (i.e. in the whole MDD group in each study); mean age of patients; illness duration; and the mean number of illness episodes. Depression symptom severity could not be explored by meta-regression due to a lack of consistent depression-scale data. The percentage of MDD patients who had ever received psychotherapy could not be explored by meta-regression because data were available for fewer than nine studies (Radua \& Mataix-Cols, 2009). As described in previous meta-analyses, in order to minimize the detection of spurious relationships, we decreased the probability threshold to 0.0005 required abnormalities to be detected both in the slope and in one of the extremes of the regressor, and discarded findings in regions other than those detected in the main analyses (Radua \& Mataix-Cols, 2009). Furthermore, regression plots were visually inspected to discard fittings driven by too few studies (Radua \& Mataix-Cols, 2009).

\section{Results}

\section{Included studies and sample characteristics}

The search strategy identified 1144 studies, of which 11 studies (Tang et al. 2007; Wagner et al. 2008; Cheng et al. 2010; Jia et al. 2010; Lai et al. 2010; Scheuerecker et al. 2010; Zou et al. 2010; Salvadore et al. 2011; Arnone et al. 2012; Ma et al. 2012; Zhang et al. 2012), including 14 datasets, ultimately met inclusion criteria, with 400 unmedicated MDD patients (142 male and 258 female; mean age 33.8 years) and 424 healthy controls (163 male and 261 female; mean age 32.4 years). Of two studies using overlapping samples, the one with the most subjects was included after email discussion with the authors (Arnone et al. 2012). Fig. 1 shows a flow diagram of the identification and attrition of studies. Table 1 summarizes clinical and demographic data from all included studies. In no study was there any significant difference in age and sex between the MDD group and the healthy controls. 


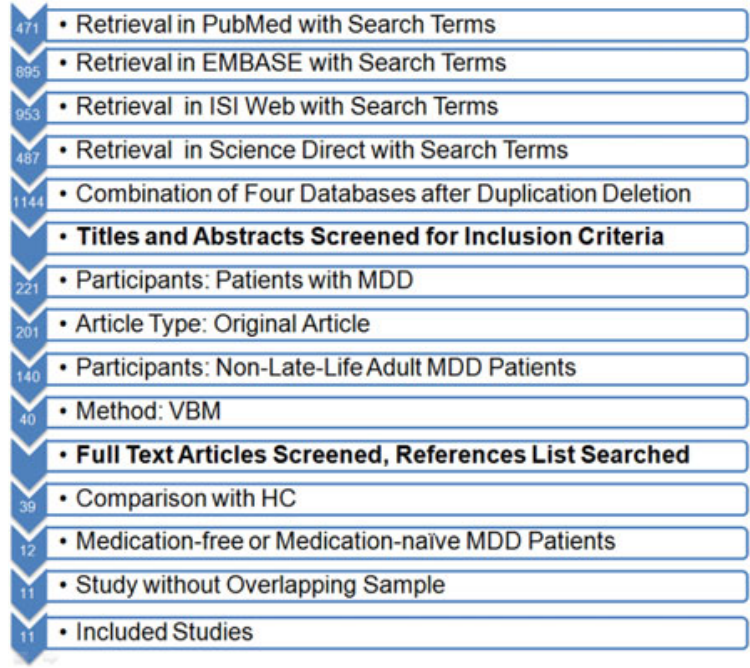

Fig. 1. Identification and attrition of studies. Search terms were ((depression OR depressive disorder OR unipolar depression) AND (voxel-based morphometry OR VBM OR voxel ${ }^{*}$ OR morphometry)). MDD, Major depressive disorder; VBM, voxel-based morphometry; HC, healthy controls.

\section{Pooled meta-analysis of all included medication-free studies}

A group comparison of MDD patients with healthy controls across the 14 datasets revealed grey matter volume reductions in the left inferior frontal gyrus, the right middle frontal gyrus extending to the inferior frontal gyrus, the right superior frontal gyrus, the left superior frontal gyrus extending to the middle frontal gyrus, and the bilateral parahippocampal gyrus and hippocampus (Table 2 and Fig. 2). No grey matter volume increases were found.

\section{Jack-knife sensitivity analysis}

Whole-brain jack-knife sensitivity analysis showed that grey matter reduction in the right middle frontal gyrus and the right superior frontal gyrus was highly replicable, being preserved throughout all 14 combinations of datasets. The results in the left inferior and superior frontal gyrus remained significant in all but one combination, those in the left parahippocampal gyrus and the hippocampus in all but two combinations, and those in the right parahippocampal gyrus and hippocampus in all but three combinations.

\section{Subgroup meta-analysis of first-episode medication-naive studies}

In six datasets recruiting first-episode medicationnaive MDD patients the subgroup analysis revealed grey matter volume increase relative to controls in the right thalamus, and decrease in the right superior and middle frontal gyrus, the right parahippocampal gyrus and hippocampus extending to the amygdala, and the left parahippocampal gyrus and hippocampus (Table 2).

\section{Subgroup meta-analysis of medication wash-out studies}

The wash-out subgroup analysis of eight datasets showed grey matter volume increase relative to controls in the bilateral anterior cingulate cortex, and decrease in the left inferior and middle frontal gyrus extending to the superior frontal gyrus, the right middle and superior frontal gyrus, and the right parahippocampal gyrus (Table 2).

\section{Meta-regression analysis}

The percentage of female patients with MDD (i.e. in the whole MDD group in each study) was negatively associated with grey matter volume in the right hippocampus $(r=0.814$, permutation-derived $p<0.0001)$ (Fig. 3). However, this result should be interpreted with some caution, being driven only by four studies (Tang et al. 2007; Wagner et al. 2008; Lai et al. 2010; Scheuerecker et al. 2010). The mean age of patients, illness duration, and the mean number of episodes were not associated with MDD-related grey matter changes, at least linearly.

\section{Discussion}

This is to our knowledge the first meta-analysis of VBM studies in medication-free MDD patients, examining how age, sex and clinical characteristics affect grey matter volumes without the interference of medication effects. The medication-free MDD patients, compared with healthy control subjects, displayed significantly and robustly reduced grey matter in the prefrontal and limbic regions. Notably, increasing percentage of female patients correlated with decreasing grey matter volumes in the right hippocampus. Although most of the results of the two subgroup meta-analyses were consistent, there were some differences. Increased grey matter volume in the right thalamus compared with controls was only seen in the first-episode medication-naive studies, which might suggest that increased right thalamus volume is a trait for MDD, i.e. directly related to MDD per se. Conversely, increased grey matter volume in the bilateral ACC compared with controls was only seen in the subgroup meta-analysis of wash-out studies, which is at least consistent with the idea that increased ACC volume is an effect of medication. To our knowledge, there are no prospective randomized controlled studies 
Table 1. Demographic and clinical characteristics of subjects in the 14 voxel-based morphometry datasets included in the meta-analysis

\begin{tabular}{|c|c|c|c|c|c|c|c|c|c|c|}
\hline \multirow[b]{2}{*}{ Study } & \multicolumn{2}{|c|}{$\begin{array}{l}\text { Subjects, } n \\
\text { (females, } n \text { ) }\end{array}$} & \multicolumn{2}{|c|}{ Age, years } & \multirow{2}{*}{$\begin{array}{l}\text { Illness } \\
\text { duration, } \\
\text { years }\end{array}$} & \multirow{2}{*}{$\begin{array}{l}\text { Severity } \\
\text { (scale type) }\end{array}$} & \multirow{2}{*}{$\begin{array}{l}\text { Mean } \\
\text { no. of } \\
\text { episodes }\end{array}$} & \multirow{2}{*}{$\begin{array}{l}\text { Fraction of } \\
\text { MDD patients } \\
\text { receiving } \\
\text { psychotherapy }\end{array}$} & \multirow{2}{*}{$\begin{array}{l}\text { Statistical } \\
\text { threshold }\end{array}$} & \multirow[b]{2}{*}{ Drug status } \\
\hline & MDD & $\mathrm{HC}$ & MDD & $\mathrm{HC}$ & & & & & & \\
\hline Arnone et al. (2012) dMDD & $39(27)$ & $66(46)$ & 36.3 & 32.1 & N.A. & $\geqslant 20$ (MADRS) & 3.3 & N.A. & FWE & Drug-naive or medication-free for 4 years (mean) \\
\hline Arnone et al. (2012) rMDD & $25(20)$ & $66(46)$ & 34.5 & 32.1 & N.A. & $\leqslant 10$ (MADRS) & 2.8 & N.A. & FWE & Drug-naive or medication-free for $>4$ months \\
\hline Cheng et al. (2010) & $68(47)$ & $68(47)$ & 29.91 & 30.54 & 0.92 & 22.31 (HAMD) & FE & 0 & Uncorrected & Drug-naive \\
\hline Jia et al. (2010) nMDD & $36(16)$ & $52(28)$ & 34.7 & 37.1 & 1.75 & 22.3 (HAMD) & N.A. & N.A. & FWE & Antidepressant-free for $>2$ weeks \\
\hline Jia et al. (2010) sMDD & $16(11)$ & $52(28)$ & 34.2 & 37.1 & 6.67 & 24.6 (HAMD) & N.A. & N.A. & FWE & Antidepressant-free for $>2$ weeks \\
\hline Lai et al. (2010) & $16(11)$ & $15(11)$ & 37.91 & 34.3 & 0.36 & 35.91 (HAMD) & FE & 0 & FWE & Drug-naive \\
\hline Ma et al. (2012) & $17(7)$ & $17(7)$ & 26.71 & 24.24 & 0.22 & 25.58 (HAMD) & $\mathrm{FE}$ & 0 & ASC & Drug-naive \\
\hline Salvadore et al. (2011) dMDD & $58(37)$ & $107(60)$ & 38.8 & 36.2 & 18.4 & 26 (MADRS) & N.A. & N.A. & FWE & Medication-free for $1-130$ months \\
\hline Salvadore et al. (2011) rMDD & $27(21)$ & $107(60)$ & 40.2 & 36.2 & 15.1 & 1.6 (MADRS) & N.A. & N.A. & FWE & Medication-free for 3-240 months \\
\hline Scheuerecker et al. (2010) & $13(3)$ & $15(5)$ & 37.9 & 35.5 & 4.36 & 20.5 (HAMD) & 1.45 & $2 / 13$ & Uncorrected & Medication free for $>1$ year \\
\hline Tang et al. (2007) & $14(14)$ & $13(13)$ & 29.5 & 29.46 & 0.45 & $\geqslant 18$ (HAMD) & FE & N.A. & Uncorrected & Drug-naive \\
\hline Wagner et al. (2008) & $15(15)$ & $16(16)$ & 41.4 & 38.8 & 7.5 & 23.5 (HAMD) & 1.4 & 0 & Uncorrected & Medication-free for 1 week (mean) \\
\hline Zhang et al. (2012) & $33(16)$ & $32(15)$ & 20.52 & 21.03 & N.A. & 37.67 (CES-D) & FE & N.A. & FWE & Drug-naive \\
\hline Zou et al. (2010) & $23(13)$ & $23(13)$ & 31.1 & 36.6 & 0.65 & 24.4 (HAMD) & FE & N.A. & Corrected & Drug-naive \\
\hline
\end{tabular}

MDD, Major depressive disorder; HC, healthy control; dMDD, currently depressed subjects; N.A., not available; MADRS, Montgomery-Asberg Depression Rating Scale; FWE, familywise error correction; rMDD, subjects currently in remission; HAMD, Hamilton Depression Rating Scale; FE, first episode; nMDD, depressed patients without a history of suicide attempts; sMDD, depressed patients with a history of suicide attempts; ASC, AlphaSim correction; CES-D, Center for Epidemiological Studies Depression Scale. 
Table 2. Regional differences in grey matter volume between MDD patients and HC subjects identified by the present meta-analyses (voxelwise $p<0.005$ and FWHM 20 mm)

\begin{tabular}{|c|c|c|c|c|c|c|c|}
\hline \multirow[b]{2}{*}{ Region } & \multicolumn{3}{|c|}{ Talairach coordinates } & \multirow{2}{*}{$\begin{array}{l}\text { SDM } \\
z \text { score }\end{array}$} & \multirow[b]{2}{*}{$p$, uncorrected } & \multirow[b]{2}{*}{ Voxels, $n$} & \multirow[b]{2}{*}{ Cluster breakdown (voxels, $n$ ) } \\
\hline & $\mathrm{x}$ & $\mathrm{y}$ & $\mathrm{z}$ & & & & \\
\hline \multicolumn{8}{|l|}{ Pooled meta-analysis of all included studies } \\
\hline \multicolumn{8}{|l|}{$\mathrm{MDD}<\mathrm{HC}$} \\
\hline Left inferior frontal gyrus, BA 45 & -50 & 24 & 18 & -1.594 & 0.000380218 & 105 & $\begin{array}{l}\text { Inferior frontal gyrus, BA 9, 44, 45, } 46 \text { (86) } \\
\text { Precentral gyrus, BA } 44 \text { (2) } \\
\text { Middle frontal gyrus, BA } 46 \text { (17) }\end{array}$ \\
\hline Left parahippocampal gyrus, hippocampus & -28 & -16 & -18 & -1.49 & 0.000721901 & 56 & $\begin{array}{l}\text { Hippocampus (32) } \\
\text { Parahippocampal gyrus, BA 28, 34, } 35 \text { (24) }\end{array}$ \\
\hline Right middle frontal gyrus, BA 46 & 52 & 42 & 16 & -1.488 & 0.000734746 & 78 & $\begin{array}{l}\text { Middle frontal gyrus, BA } 46(58) \\
\text { Inferior frontal gyrus, BA } 46 \text { (20) }\end{array}$ \\
\hline Right superior frontal gyrus, BA 9 & 40 & 40 & 30 & -1.487 & 0.000757868 & 45 & $\begin{array}{l}\text { Superior frontal gyrus, BA } 9 \text { (36) } \\
\text { Middle frontal gyrus, BA } 9 \text { (9) }\end{array}$ \\
\hline Left superior frontal gyrus, BA 9 & -36 & 36 & 32 & -1.327 & 0.002019268 & 37 & $\begin{array}{l}\text { Superior frontal gyrus, BA } 9 \text { (13) } \\
\text { Middle frontal gyrus, BA } 9 \text { (24) }\end{array}$ \\
\hline Right parahippocampal gyrus, hippocampus & 24 & -18 & -20 & -1.31 & 0.002245344 & 27 & $\begin{array}{l}\text { Parahippocampal gyrus, BA 28, } 35 \text { (18) } \\
\text { Hippocampus (9) }\end{array}$ \\
\hline \multicolumn{8}{|l|}{ Subgroup meta-analysis of medication-naive studies } \\
\hline Right thalamus & 22 & -26 & 12 & 1.063 & 0.000184971 & 391 & $\begin{array}{l}\text { Thalamus (351) } \\
\text { Caudate (31) } \\
\text { Parahippocampal gyrus, BA } 30 \text { (8) } \\
\text { Hippocampus (1) }\end{array}$ \\
\hline \multicolumn{8}{|l|}{$\mathrm{MDD}<\mathrm{HC}$} \\
\hline Right superior frontal gyrus, BA 6 & 16 & -8 & 66 & -1.779 & 0.000292871 & 71 & Superior frontal gyrus, BA 6 (71) \\
\hline Right middle frontal gyrus, BA 6 & 22 & -6 & 58 & -1.779 & 0.000292871 & 66 & Middle frontal gyrus, BA 6 (66) \\
\hline Right parahippocampal gyrus, amygdala & 24 & -2 & -12 & -1.362 & 0.001662171 & 217 & $\begin{array}{l}\text { Amygdala (94) } \\
\text { Lentiform nucleus (76) } \\
\text { Parahippocampal gyrus, BA34, } 28 \text { (31) } \\
\text { Subcallosal gyrus, BA } 34 \text { (16) }\end{array}$ \\
\hline Left parahippocampal gyrus, hippocampus & -20 & -20 & -12 & -1.037 & 0.003722543 & 22 & $\begin{array}{l}\text { Parahippocampal gyrus, BA 28, } 35 \text { (21) } \\
\text { Hippocampus (1) }\end{array}$ \\
\hline
\end{tabular}




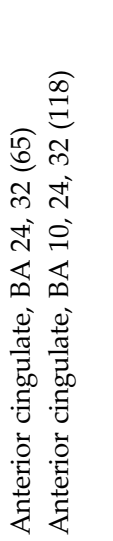
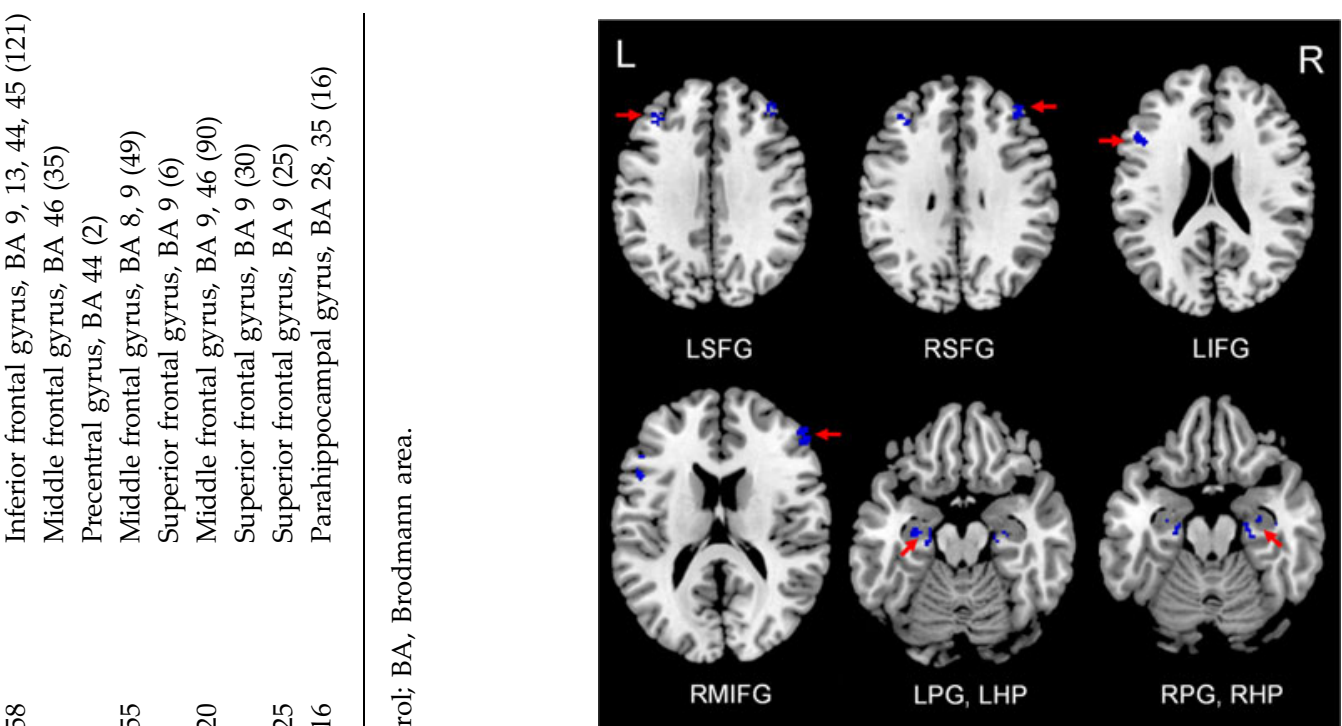

Fig. 2. Regions showing reduced grey matter in medication-free major depressive disorder patients compared with healthy controls. L, Left; R, right; SFG, superior frontal gyrus; IFG, inferior frontal gyrus; MIFG, middle frontal gyrus; PG, parahippocampal gyrus; HP, hippocampus.

of the long-term effects of antidepressants on brain structure, and this finding in the ACC merits further investigation. This result differs from that of previous meta-analyses which showed decreased grey matter volume in the bilateral ACC (Bora et al. 2012; Du et al. 2012; Lai, 2013) and decreased grey matter in the left thalamus (Du et al. 2012), compared with controls, which might be explained by the fact that all of these studies included both medicated and unmedicated MDD patients.

\section{Findings in the hippocampus and parahippocampus}

The reduction in grey matter volume in the bilateral hippocampus and parahippocampal gyrus in medication-free MDD patients, compared with controls, which we identified in the present study is consistent with a recent meta-analysis of traditional MRI and computed tomography studies on depression (Kempton et al. 2011). The hippocampus and parahippocampus are key regions of the limbic system that play a central role in the regulation of emotions, motivation, memory and affective dimension of pain (Fang et al. 2009; Fossati, 2012); they are extensively connected with other cortical and subcortical structures in the frontal and temporal lobes and play an important role in cognitive processes of MDD (Price \& Drevets, 2010). Thus grey matter damage in these regions may be related to memory function impairment and emotion processing, which is the main 

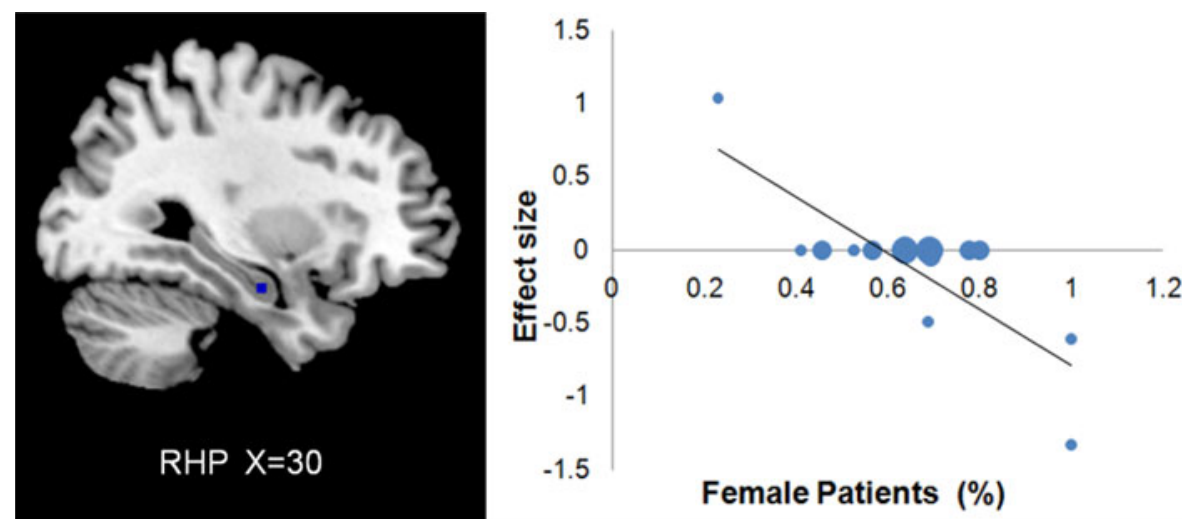

Fig. 3. Meta-regression results show that the percentage of female major depressive disorder patients is negatively correlated with grey matter in the right hippocampus (RHP). In the graphs, the effect sizes needed to create this plot have been extracted from the peak of maximum slope significance, and each study is represented as a dot, whose size reflects sample size. Large dots indicate samples with $>40$ patients; medium dots, $20-40$ patients; and small dots, $<20$ patients. The regression line (meta-regression signed differential mapping slope) is shown.

clinical manifestation of MDD. Furthermore, in a 3-year longitudinal study, hippocampal grey matter volume declined significantly more in MDD patients than in controls, and also significantly more in patients who did not remit during the period than those who did, suggesting that depression may lead to changes in hippocampal morphologic structure (Frodl et al. 2008).

\section{Findings in the prefrontal cortex}

Our finding of decreased grey matter in medicationfree MDD patients in the superior, middle and inferior frontal gyrus compared with controls corresponds to the right DLPFC and left DLPFC and ventrolateral prefrontal cortex. Postmortem studies have demonstrated reductions in glial cell density and neuronal size in the deeper cortical layers of the PFC in MDD which may underlie these grey matter abnormalities detected by neuroimaging (Rajkowska et al. 1999; Rajkowska, 2000; Cotter et al. 2002). Furthermore, there is evidence that increased glutamatergic transmission in depression may cause excitotoxic damage and cellular loss, dendritic reshaping and consequent grey matter morphometric abnormalities (Price \& Drevets, 2010). The lateral prefrontal lobe has been theoretically identified as important in the pathophysiology of MDD, being associated with cognition dysfunction and emotion regulation in depression (Levesque et al. 2003; Ochsner et al. 2004; Phan et al. 2005; Matsuo et al. 2007; Walter et al. 2007; Price \& Drevets, 2010). Moreover a recent longitudinal therapeutic study on SSRI-treated MDD patients has shown grey matter changes in the left DLPFC, which is associated with top-down neuronal modulation of emotion (Smith et al. 2013); a defect in the regulation of negative affect due to DLPFC dysfunction is a plausible mechanism for DLPFC involvement in depression (Koenigs \& Grafman, 2009). Therefore, the identified grey matter abnormality in the prefrontal gyrus may be part of the structural underpinning of the prefrontal dysfunction in MDD patients.

\section{Sex effects}

The percentage of female MDD patients was negatively correlated with grey matter volume in the right hippocampus, which indicates that female medicationfree MDD patients tend to have smaller right hippocampus. Other structural imaging studies have shown significant sex effects consistent with the present results: greater hippocampal volume in male compared with female MDD patients (Steffens et al. 2000; Frodl et al. 2002; Kronmuller et al. 2009), and specifically greater right hippocampal volumes in male than female psychotropic-naive patients with familial MDD (MacMaster et al. 2008). Sex differences are seen in epidemiological and clinical aspects of MDD: rates of MDD are higher in females than males (Kessler et al. 2005); most studies report greater sensitivity in women to the depressogenic effect of stressful life events (Post et al. 1995); female MDD patients show higher rates of suicide attempts and co-morbid anxiety disorders, while male MDD patients are more likely to show psychomotor agitation and have co-morbid substance abuse disorders (Roeloffs et al. 2001; Marcus et al. 2005). The sex difference in right hippocampus volume in MDD may contribute to differences in epidemiological and clinical aspects of depression. However, other reports (Vakili et al. 2000; MacQueen et al. 2003; Neumeister et al. 2005) found no such sex difference. The heterogeneous findings of structural 
imaging studies can perhaps be explained by the highly variable female:male ratio among studies.

\section{Limitations and conclusions}

This study has several limitations. First, peak-based meta-analyses are based on summarized (i.e. coordinates from published studies) rather than raw statistical brain maps, and this approach may result in less accurate results (Nakao et al. 2011). Second, the main meta-analysis included primary studies enrolling medicated MDD patients who underwent a wash-out period before MRI scanning and the results of the two subgroups were to some extent different. Clearly the best way to minimize the effects of illness duration and antidepressant treatment is to focus on drug-naive MDD patients. However, the sample size of our drugnaive subgroup analysis is necessarily small, which limits the generalizability of the results. Meta-analyses of first-episode drug-naive MDD are needed when the number of published VBM studies on such patients permits. Third, the meta-regression finding that the percentage of female MDD patients was negatively correlated with grey matter volume in the right hippocampus was driven by only four studies.

In summary, our findings indicate that medicationfree MDD patients have significantly and robustly reduced grey matter mainly in prefrontal and limbic brain regions excluding the confounding influences of medication status. The subgroup meta-analysis results suggest that increased right thalamus volume may be directly related to MDD, while increased ACC volume may be an effect of medication. Metaregression results suggest that female medication-free MDD patients may have smaller right hippocampus, which may constitute the structural underpinnings of the sex differences observed in both epidemiological and clinical aspects of MDD. Future studies will benefit from the use of a longitudinal approach to track patients from disease onset and through followup and examine the dynamic effect of medication on brain structure in MDD patients. Longitudinal studies that investigate the dynamic brain structure change of competent MDD patients who reject all therapies are also encouraged.

\section{Acknowledgements}

This work was supported by the National Natural Science Foundation (grant nos. 81030027, 81227002 and 81220108013), the National Key Technologies R\&D Programme (programme no. 2012BAI01B03), and the Programme for Changjiang Scholars and Innovative Research Team in University (PCSIRT, grant no. IRT1272) of China.

\section{Declaration of Interest}

None.

\section{References}

Arnone D, McKie S, Elliott R, Juhasz G, Thomas EJ, Downey D, Williams S, Deakin JF, Anderson IM (2012). State-dependent changes in hippocampal grey matter in depression. Molecular Psychiatry 6, 1265-1272.

Ashburner J, Friston KJ (2001). Why voxel-based morphometry should be used. NeuroImage 14, 1238-1243.

Banasr M, Soumier A, Hery M, Mocaer E, Daszuta A (2006) Agomelatine, a new antidepressant, induces regional changes in hippocampal neurogenesis. Biological Psychiatry 59, 1087-1096.

Blease C (2011). Deception as treatment: the case of depression. Journal of Medical Ethics 37, 13-16.

Bora E, Fornito A, Pantelis C, Yucel M (2012). Gray matter abnormalities in major depressive disorder: a meta-analysis of voxel based morphometry studies. Journal of Affective Disorders 138, 9-18.

Bora E, Fornito A, Radua J, Walterfang M, Seal M, Wood SJ, Yucel M, Velakoulis D, Pantelis C (2011). Neuroanatomical abnormalities in schizophrenia: a multimodal voxelwise meta-analysis and meta-regression analysis. Schizophrenia Research 127, 46-57.

Brambilla P, Nicoletti MA, Harenski K, Sassi RB, Mallinger AG, Frank E, Kupfer DJ, Keshavan MS, Soares JC (2002). Anatomical MRI study of subgenual prefrontal cortex in bipolar and unipolar subjects. Neuropsychopharmacology 27, 792-799.

Chang K, Karchemskiy A, Barnea-Goraly N, Garrett A, Simeonova DI, Reiss A (2005). Reduced amygdalar gray matter volume in familial pediatric bipolar disorder. Journal of the American Academy of Child and Adolescent Psychiatry 44, 565-573.

Cheng YQ, Xu J, Chai P, Li HJ, Luo CR, Yang T, Li L, Shan BC, Xu XF, Xu L (2010). Brain volume alteration and the correlations with the clinical characteristics in drug-naïve first-episode MDD patients: a voxel-based morphometry study. Neuroscience Letters 480, 30-34.

Cotter D, Mackay D, Chana G, Beasley C, Landau S, Everall IP (2002). Reduced neuronal size and glial cell density in area 9 of the dorsolateral prefrontal cortex in subjects with major depressive disorder. Cerebral Cortex 12, 386-394.

Czeh B, Michaelis T, Watanabe T, Frahm J, de Biurrun G, van Kampen M, Bartolomucci A, Fuchs E (2001). Stress-induced changes in cerebral metabolites, hippocampal volume, and cell proliferation are prevented by antidepressant treatment with tianeptine. Proceedings of the National Academy of Sciences USA 98, 12796-12801.

Davies RR, Scahill V, Graham A, Williams G, Graham K, Hodges J (2009). Development of an MRI rating scale for multiple brain regions: comparison with volumetrics and with voxel-based morphometry. Neuroradiology 51, 491-503.

Du MY, Wu QZ, Yue Q, Li J, Liao Y, Kuang WH, Huang XQ, Chan RC, Mechelli A, Gong QY (2012). Voxelwise meta-analysis of gray matter reduction in major depressive 
disorder. Progress in Neuro-Psychopharmacology and Biological Psychiatry 36, 11-16.

Duman RS, Monteggia LM (2006). A neurotrophic model for stress-related mood disorders. Biological Psychiatry 59, 1116-1127.

Fang J, Jin Z, Wang Y, Li K, Kong J, Nixon EE, Zeng Y, Ren Y, Tong H, Wang P, Hui KK (2009). The salient characteristics of the central effects of acupuncture needling: limbic-paralimbic-neocortical network modulation. Human Brain Mapping 30, 1196-1206.

Fossati P (2012). Neural correlates of emotion processing: from emotional to social brain. European Neuropsychopharmacology 22, S487-S491.

Frodl T, Meisenzahl EM, Zetzsche T, Born C, Groll C, Jager M, Leinsinger G, Bottlender R, Hahn K, Moller HJ (2002). Hippocampal changes in patients with a first episode of major depression. American Journal of Psychiatry 159, 1112-1118.

Frodl TS, Koutsouleris N, Bottlender R, Born C, Jager M, Scupin I, Reiser M, Moller HJ, Meisenzahl EM (2008). Depression-related variation in brain morphology over 3 years: effects of stress? Archives of General Psychiatry 65, 1156-1165.

Hamilton JP, Siemer M, Gotlib IH (2008). Amygdala volume in major depressive disorder: a meta-analysis of magnetic resonance imaging studies. Molecular Psychiatry 13, 993-1000.

Hasler G (2010). Pathophysiology of depression: do we have any solid evidence of interest to clinicians? World Psychiatry 9, 155-161.

Jia Z, Huang $X$, Wu Q, Zhang T, Lui S, Zhang J, Amatya N, Kuang W, Chan RC, Kemp GJ, Mechelli A, Gong $Q$ (2010). High-field magnetic resonance imaging of suicidality in patients with major depressive disorder. American Journal of Psychiatry 167, 1381-1390.

Kempton MJ, Salvador Z, Munafo MR, Geddes JR, Simmons A, Frangou S, Williams SC (2011). Structural neuroimaging studies in major depressive disorder. Meta-analysis and comparison with bipolar disorder. Archives of General Psychiatry 68, 675-690.

Kessler RC, Chiu WT, Demler O, Merikangas KR, Walters EE (2005). Prevalence, severity, and comorbidity of 12-month DSM-IV disorders in the National Comorbidity Survey Replication. Archives of General Psychiatry 62, 617-627.

Koenigs M, Grafman J (2009). The functional neuroanatomy of depression: distinct roles for ventromedial and dorsolateral prefrontal cortex. Behavioural Brain Research 201, 239-243.

Kronmuller KT, Schroder J, Kohler S, Gotz B, Victor D, Unger J, Giesel F, Magnotta V, Mundt C, Essig M, Pantel J (2009). Hippocampal volume in first episode and recurrent depression. Psychiatry Research 174, 62-66.

Lai CH (2013). Gray matter volume in major depressive disorder: a meta-analysis of voxel-based morphometry studies. Psychiatry Research 211, 37-46.

Lai CH, Hsu YY, Wu YT (2010). First episode drug-naïve major depressive disorder with panic disorder: gray matter deficits in limbic and default network structures. European Neuropsychopharmacology 20, 676-682.

Lavretsky H, Roybal DJ, Ballmaier M, Toga AW, Kumar A (2005). Antidepressant exposure may protect against decrement in frontal gray matter volumes in geriatric depression. Journal of Clinical Psychiatry 66, 964-967.

Levesque J, Eugene F, Joanette Y, Paquette V, Mensour B, Beaudoin G, Leroux JM, Bourgouin P, Beauregard $M$ (2003). Neural circuitry underlying voluntary suppression of sadness. Biological Psychiatry 53, 502-510.

Ma C, Ding J, Li J, Guo W, Long Z, Liu F, Gao Q, Zeng L, Zhao J, Chen H (2012). Resting-state functional connectivity bias of middle temporal gyrus and caudate with altered gray matter volume in major depression. PLOS ONE 7, 24.

MacMaster FP, Mirza Y, Szeszko PR, Kmiecik LE, Easter PC, Taormina SP, Lynch M, Rose M, Moore GJ, Rosenberg DR (2008). Amygdala and hippocampal volumes in familial early onset major depressive disorder. Biological Psychiatry 63, 385-390.

MacQueen GM, Campbell S, McEwen BS, Macdonald K, Amano S, Joffe RT, Nahmias C, Young LT (2003). Course of illness, hippocampal function, and hippocampal volume in major depression. Proceedings of the National Academy of Sciences USA 100, 1387-1392.

Marcus SM, Young EA, Kerber KB, Kornstein S, Farabaugh AH, Mitchell J, Wisniewski SR, Balasubramani GK, Trivedi MH, Rush AJ (2005). Gender differences in depression: findings from the STAR ${ }^{*} \mathrm{D}$ study. Journal of Affective Disorders 87, 141-150.

Mathers C, Fat DM, Boerma J (2008). The Global Burden of Disease: 2004 Update. World Health Organization: Geneva.

Matsuo K, Glahn DC, Peluso MA, Hatch JP, Monkul ES, Najt P, Sanches M, Zamarripa F, Li J, Lancaster JL, Fox PT, Gao JH, Soares JC (2007). Prefrontal hyperactivation during working memory task in untreated individuals with major depressive disorder. Molecular Psychiatry 12, 158-166.

Nakao T, Radua J, Rubia K, Mataix-Cols D (2011). Gray matter volume abnormalities in ADHD: voxel-based meta-analysis exploring the effects of age and stimulant medication. American Journal of Psychiatry 168, 1154-1163.

Neumeister A, Wood S, Bonne O, Nugent AC, Luckenbaugh DA, Young T, Bain EE, Charney DS, Drevets WC (2005). Reduced hippocampal volume in unmedicated, remitted patients with major depression versus control subjects. Biological Psychiatry 57, 935-937.

Ochsner KN, Ray RD, Cooper JC, Robertson ER, Chopra S, Gabrieli JD, Gross JJ (2004). For better or for worse: neural systems supporting the cognitive down- and up-regulation of negative emotion. NeuroImage 23, 483-499.

Palaniyappan L, Balain V, Radua J, Liddle PF (2012). Structural correlates of auditory hallucinations in schizophrenia: a meta-analysis. Schizophrenia Research 137, 169-173.

Phan KL, Fitzgerald DA, Nathan PJ, Moore GJ, Uhde TW, Tancer ME (2005). Neural substrates for voluntary suppression of negative affect: a functional magnetic resonance imaging study. Biological Psychiatry 57, 210-219. 
Post RM, Weiss SR, Smith M, Rosen J, Frye M (1995). Stress, conditioning, and the temporal aspects of affective disorders. Annals of the New York Academy of Sciences 771, 677-696.

Price JL, Drevets WC (2010). Neurocircuitry of mood disorders. Neuropsychopharmacology 35, 192-216.

Radua J, Borgwardt S, Crescini A, Mataix-Cols D, Meyer-Lindenberg A, McGuire PK, Fusar-Poli P (2012a). Multimodal meta-analysis of structural and functional brain changes in first episode psychosis and the effects of antipsychotic medication. Neuroscience and Biobehavioral Reviews 36, 2325-2333.

Radua J, Mataix-Cols D (2009). Voxel-wise meta-analysis of grey matter changes in obsessive-compulsive disorder. British Journal of Psychiatry 195, 393-402.

Radua J, Mataix-Cols D, Phillips ML, El-Hage W, Kronhaus DM, Cardoner N, Surguladze S (2012b). A new meta-analytic method for neuroimaging studies that combines reported peak coordinates and statistical parametric maps. European Psychiatry 27, 605-611.

Radua J, Via E, Catani M, Mataix-Cols D (2011). Voxel-based meta-analysis of regional white-matter volume differences in autism spectrum disorder versus healthy controls. Psychological Medicine 41, 1539-1550.

Rajkowska G (2000). Postmortem studies in mood disorders indicate altered numbers of neurons and glial cells. Biological Psychiatry 48, 766-777.

Rajkowska G, Miguel-Hidalgo JJ, Wei J, Dilley G, Pittman SD, Meltzer HY, Overholser JC, Roth BL, Stockmeier CA (1999). Morphometric evidence for neuronal and glial prefrontal cell pathology in major depression. Biological Psychiatry 45, 1085-1098.

Rizvi SJ, Kennedy SH (2012). Emerging drugs for major depressive disorder: an update. Expert Opinion on Emerging Drugs 17, 285-295.

Roeloffs CA, Fink A, Unutzer J, Tang L, Wells KB (2001). Problematic substance use, depressive symptoms, and gender in primary care. Psychiatric Services 52, 1251-1253.

Salvadore G, Nugent AC, Lemaitre H, Luckenbaugh DA, Tinsley R, Cannon DM, Neumeister A, Zarate CA Jr, Drevets WC (2011). Prefrontal cortical abnormalities in currently depressed versus currently remitted patients with major depressive disorder. Neurolmage 54, 2643-2651.

Savitz J, Nugent AC, Bogers W, Liu A, Sills R, Luckenbaugh DA, Bain EE, Price JL, Zarate C, Manji HK, Cannon DM, Marrett S, Charney DS, Drevets WC (2010). Amygdala volume in depressed patients with bipolar disorder assessed using high resolution 3 T MRI: the impact of medication. Neurolmage 49, 2966-2976.

Scheuerecker J, Meisenzahl EM, Koutsouleris N, Roesner M, Schopf V, Linn J, Wiesmann M, Bruckmann H, Moller HJ, Frodl T (2010). Orbitofrontal volume reductions during emotion recognition in patients with major depression. Journal of Psychiatry and Neuroscience 35, 311-320.

Smith R, Chen K, Baxter L, Fort C, Lane RD (2013). Antidepressant effects of sertraline associated with volume increases in dorsolateral prefrontal cortex. Journal of Affective Disorders 146, 414-419.

Steffens DC, Byrum CE, McQuoid DR, Greenberg DL, Payne ME, Blitchington TF, MacFall JR, Krishnan KR (2000). Hippocampal volume in geriatric depression. Biological Psychiatry 48, 301-309.

Tang Y, Wang F, Xie G, Liu J, Li L, Su L, Liu Y, Hu X, He Z, Blumberg HP (2007). Reduced ventral anterior cingulate and amygdala volumes in medication-naïve females with major depressive disorder: a voxel-based morphometric magnetic resonance imaging study. Psychiatry Research 156, 83-86.

Vakili K, Pillay SS, Lafer B, Fava M, Renshaw PF, Bonello-Cintron CM, Yurgelun-Todd DA (2000). Hippocampal volume in primary unipolar major depression: a magnetic resonance imaging study. Biological Psychiatry 47, 1087-1090.

Wagner G, Koch K, Schachtzabel C, Reichenbach JR, Sauer H, Schlösser RG (2008). Enhanced rostral anterior cingulate cortex activation during cognitive control is related to orbitofrontal volume reduction in unipolar depression. Journal of Psychiatry and Neuroscience 33, 199-208.

Walter H, Wolf RC, Spitzer M, Vasic N (2007). Increased left prefrontal activation in patients with unipolar depression: an event-related, parametric, performance-controlled fMRI study. Journal of Affective Disorders 101, 175-185.

Zhang X, Yao S, Zhu X, Wang X, Zhong M (2012). Gray matter volume abnormalities in individuals with cognitive vulnerability to depression: a voxel-based morphometry study. Journal of Affective Disorders 136, 443-452.

Zou K, Deng W, Li T, Zhang B, Jiang L, Huang C, Sun X (2010). Changes of brain morphometry in first-episode, drug-naive, non-late-life adult patients with major depression: an optimized voxel-based morphometry study. Biological Psychiatry 67, 186-188. 